\title{
Manejo anestésico de paciente con enfermedad de Moyamoya sometido a encefaloduroarteriomiosinangiosis
}

\author{
Ana M. ${ }^{a}$ Pérez-Muñoz ${ }^{1 *}$, Ana Martínez-Saniger ${ }^{2}$, Mercedes Echevarría-Moreno ${ }^{1}$ e Ignacio Jiménez-López ${ }^{2}$ \\ ${ }^{1}$ Anestesiología y Reanimación, Hospital Universitario Nuestra Señora de Valme; ${ }^{2}$ Anestesiología y Reanimación Hospital Universitario Virgen del \\ Rocío. Sevilla, España
}

\begin{abstract}
Resumen
La enfermedad de Moyamoya es una patología vascular infrecuente, pero de importantes implicaciones fisiopatológicas. Al estenosar los principales vasos cerebrales, origina una pérdida de la autorregulación, con riesgo elevado de provocar hipoxia en los territorios que irrigan y, por otro lado, la generación de neovascularización implica una elevada probabilidad de ruptura y hemorragia intracerebral. Por tanto, los pacientes cursan con eventos cerebrovasculares que provocan déficit neurológico. Uno de los tratamientos más extendidos consiste en el implante de la arteria temporal superficial en la región cortical afecta (encefaloduroarteriomiosinangiosis). La técnica anestésica más adecuada, así como las estrategias para optimizar la perfusión cerebral siguen en discusión. A continuación exponemos nuestra experiencia ante un paciente con afectación moderada sometido a dicho procedimiento bajo anestesia general total intravenosa con preservación del estado neurológico tras educción y adecuada evolución clínica posoperatoria.
\end{abstract}

Palabras clave: Moyamoya. Anestesia. Encefaloduroarteriomiosinangiosis.

\section{Anesthetic management of a patient with Moyamoya disease scheduled for encephaloduralarterialsynangiosis}

\begin{abstract}
Moyamoya's disease is an unusual vascular entity with important physiopathological implications. Vascular stenosis in main cerebral arteries causes a loss in self-regulation, with high risk of hypoxia. It generates neovascularization too, that implies a high probability of rupture and intracerebral hemorrhage. One of the most extended therapy is the encephalo-dural-arterial synangiosis. This treatment consists of implanting superficial temporal artery in the cortical region affects. The most appropriate anesthetic technique as well as the strategies to optimize cerebral perfusion are still under discussion. In this article we present our experience with a patient undergoing this procedure under total intravenous anesthesia, with preservation of neurological status after extubation.
\end{abstract}

Key words: Moyamoya. Anesthesia. Encephaloduralarterialsynangiosis.

Correspondencia:

Ana M. ${ }^{a}$ Pérez-Muñoz

E-mail: nitapemu@gmail.com
Disponible en internet: $10-10-2018$ Rev Argent Anestesiol. 2018;76(2):85-88 www.revistaargentinaanestesiologia.com 0370-7792 @ 2018 Federación Argentina de Asociaciones, Anestesia, Analgesia y Reanimación. Publicado por Permanyer México SA de CV. Este es un artículo Open Access bajo la licencia CC BY-NC-ND (http://creativecommons.org/licenses/by-nc-nd/4.0/). 


\section{Introducción}

La enfermedad de Moyamoya (EMM) es una patología cerebrovascular poco frecuente que provoca la estenosis progresiva de las principales arterias del polígono de Willis. En 1957, Takeuchi y Shimizui la describieron por primera vez y la designaron basándose en el parecido de las imágenes vasculares dibujadas en la angiografía cerebral con las «bocanadas de humo de los cigarros en el aire»" 1 .

Existe una clara predisposición étnica y familiar. Es más frecuente en países asiáticos, y alcanza su máxima prevalencia en Japón (6 por 100,000 habitantes); no obstante, también está presente en América o Europa, como en nuestro propio medio, con una prevalencia de 1.21 por 100,000 habitantes ${ }^{2}$. También destaca una mayor afectación en el sexo femenino, con una tasa 1.8:1. En su etiología están implicados múltiples genes localizados en diferentes cromosomas, con herencia autosómica dominante, con baja penetrancia y factores sistémicos aún por definir.

Los factores encargados de su fisiopatología se desconocen. Generalmente afecta a la porción distal de las arterias carótidas internas y la región proximal de las cerebrales medias y anteriores, y es menos frecuente encontrar alteraciones en la cerebral posterior. La lesión principal consiste en la estenosis concéntrica, que puede progresar hasta ocasionar la oclusión completa, y que favorece la neovascularización colateral, responsable de las imágenes angiográficas características ${ }^{3}$. Los vasos afectados por la EMM han perdido la capacidad de autorregulación, por lo que generan hipoxia en los tejidos que irrigan. Los factores humorales liberados por dichas regiones ocasionan el desarrollo de la circulación colateral (Tabla 1).

Los síntomas suelen aparecer en dos rangos de edad: niños de entre 3 a 10 años y adultos entre 35 y 45 años. Característicamente se presentan como accidentes isquémicos transitorios, ictus isquémicos y hemorragias intracraneales. La cefalea o las crisis epilépticas son menos frecuentes. Existen algunos factores desencadenantes, como la tos, el llanto o el ejercicio físico intenso, y se sabe que en los niños implica un deterioro neurológico progresivo. Dos tercios de los pacientes presentarán una recidiva en menos de 5 años desde su debut, independientemente de si la afectación vascular es uni o bilateral ${ }^{3,4}$.

El gold standard para el diagnóstico sigue siendo la angiografía cerebral, aunque la angiografía por tomografía computarizada o la angiografía por resonancia magnética nuclear también pueden ser muy útiles.
El tratamiento médico de la EMM, basado en calcioantagonistas con efecto vasodilatador y antiagregantes, no frena el progreso de la enfermedad. Por otro lado, aunque existen múltiples alternativas quirúrgicas, ninguna de ellas asegura la revascularización completa del tejido cerebral comprometido. Una de las más implementadas es la encefaloduroarteriomiosinangiosis, que consiste en implantar la arteria temporal superficial sobre la región cerebral afecta para promover la angiogénesis ${ }^{1,3}$.

En la bibliografía actual existe gran controversia sobre la técnica anestésica ideal para los pacientes con EMM durante la encefaloduroarteriomiosinangiosis, por ello consideramos de interés describir nuestra experiencia en un paciente sometido a dicha intervención bajo anestesia total intravenosa (TIVA).

\section{Caso clínico}

Se trata de un varón de 51 años, originario de China, en cuyos antecedentes familiares destaca la muerte de su madre y de su hermano por ictus isquémico. El paciente es fumador de 15 cigarrillos al día, padece hipertensión arterial, en buen control con enalapril, y dislipemia, en tratamiento con simvastatina. En noviembre de 2016 presentó un episodio de hemorragia subaracnoidea y hematoma subdural temporal con secuela de afasia nominativa, y fue diagnosticado de EMM en estadio 3. Desde entonces recibe ácido acetilsalicílico $100 \mathrm{mg} / 24 \mathrm{~h}$.

Con respecto a la exploración física, destacar un índice de masa corporal de $25.7 \mathrm{~kg} / \mathrm{m}^{2}$ y la existencia de predicadores de vía aérea difícil (Test de Mallampati grado 2, apertura oral $4.5 \mathrm{~cm}$, distancia tiromentoniana (DTM) $<6 \mathrm{~cm}$ ).

Una vez en el quirófano se llevó a cabo una monitorización estándar con electrocardiografía, pulsioximetría, índice biespectral (BIS), capnografía y presión arterial $(\mathrm{PA})$ no invasiva y se canalizó una vía periférica del calibre $18 \mathrm{G}$. Tras 5 minutos de preoxigenación mediante mascarilla facial y fracción inspirada de oxígeno $\left(\mathrm{FiO}_{2}\right) \quad 100 \%$, se indujo anestesia general con fentanilo $300 \mathrm{mcg}$, propofol $170 \mathrm{mg}$, lidocaína $50 \mathrm{mg}$ y rocuronio $50 \mathrm{mg}$. La intubación endotraqueal fue dificultosa, y en la laringoscopia directa se observó un grado 3 de Cormack-Lehanne, no consiguiéndose la visualización de la glotis mediante la maniobra de BURP (backward upward rightward pressure; maniobra que se utilizada para desplazar cartílago tiroides durante la intubación ortraqueal, mejorando la visualización de la glotis). Finalmente se consiguió intubación orotraqueal (IOT) en un segundo intento mediante videolaringoscopia. A continuación se canalizó la arteria 
Tabla 1. Clasificación de los grados de la enfermedad de Moyamoya según Suzuky, 1969

\begin{tabular}{|l|l|}
\hline GRADO & DEFINICION \\
\hline I & Estenosis carótida interna terminal. \\
\hline II & Inicio de los ramos colaterales Moyamoya. \\
\hline III & $\begin{array}{l}\text { Esteosis progresiva de la carótida con } \\
\text { intensificación de los vasos Moyamoya. }\end{array}$ \\
\hline IV & $\begin{array}{l}\text { Desarrollo de colaterales desde la arteria carótida } \\
\text { externa. }\end{array}$ \\
\hline V & $\begin{array}{l}\text { Intensificación de las colaterales desde la carótida } \\
\text { externa con reducción de los vasos Moyamota. }\end{array}$ \\
\hline VI & $\begin{array}{l}\text { Oclusión total de la artería carótida interna y } \\
\text { desaparición de las colaterales Moyamoya. }\end{array}$ \\
\hline
\end{tabular}

radial para la monitorización invasiva de la PA y controles gasométricos, y vía central de acceso periférico para fluidoterapia o infusión de fármacos vasoactivos en caso de necesidad.

El mantenimiento se llevó a cabo con perfusión continua de remifentanilo, variando la dosis entre 0.05 $0.25 \mathrm{mcg} / \mathrm{kg} / \mathrm{min}$ según la respuesta hemodinámica al dolor. Con respecto al propofol, se administró siguiendo el modelo de Marsh para target control infussion (TCl), con una concentración plasmática objetivo que osciló entre $2-3 \mathrm{mcg} / \mathrm{ml}$ para niveles de índice biespectral (BIS) de 40-60.

El objetivo hemodinámico fue mantener la PA media (PAM) con variaciones máximas de un $20 \%$ con respecto a la basal, asegurando así la oxigenación del tejido cerebral irrigado por los vasos con EMM. Para ello se mantuvo una adecuada fluidoterapia con Ringer simple a $14 \mathrm{ml} / \mathrm{kg} / \mathrm{h}$ y administración de fenilefrina. Comenzamos con bolos de 50 y $100 \mathrm{mcg}$ cada 15 minutos, pero al observar la transitoriedad de los efectos, decidimos iniciar una perfusión continua con dosis de entre $0.15-0.75 \mathrm{mcg} / \mathrm{kg} / \mathrm{min}$, variando según la REMY Pespecto a la ventilación, la paciente mantuvo un nivel de dióxido de carbono al final de la espiración $\left(\mathrm{EtCO}_{2}\right)$ en torno a $28-32 \mathrm{mmHg}$, confirmada por gasometrías arteriales seriadas donde la presión parcial de dióxido de carbono en sangre arterial $\left(\mathrm{PaCO}_{2}\right)$ no superó $35 \mathrm{mmHg}$. La diuresis media rondó los $4.5 \mathrm{ml} / \mathrm{kg} / \mathrm{h}$ y se mantuvo al paciente en normotermia mediante una manta térmica. La duración total de la intervención fue de 5 horas y tanto la educción como la extubación transcurrieron sin incidentes en quirófano.

Previo traslado a la Unidad de Cuidados Intensivos (UCl) se llevó a cabo una nueva exploración neurológica, sin cambios con respecto a la basal. Durante su estancia en la $\mathrm{UCl}$, el paciente permaneció estable hemodinámica, respiratoria y neurológicamente, y a las $24 \mathrm{~h}$ de su ingresó pasó a planta de neurocirugía. En planta de hospitalización permaneció durante $48 \mathrm{~h}$ más, sin incidencias, por lo que se procedió al alta a domicilio.

\section{Discusión}

La EMM supone un reto para el neuroanestesiólogo, dado que los vasos afectos han perdido la capacidad de autorregulación, por lo que mínimas variaciones de la PAM pueden modificar la perfusión cerebral. Basándose en esto se debe realizar un planteamiento anestésico óptimo, teniendo en cuenta los efectos que cada fármaco tiene a nivel del flujo sanguíneo cerebral (FSC), la presión intracraneal (PIC) y la afectación hemodinámica, garantizando en todo momento una adecuada presión de perfusión cerebral (PPC).

Por el momento no existe evidencia científica suficiente para recomendar una técnica anestésica sobre otra. Es conocido que los hipnóticos halogenados provocan un descenso del consumo de oxígeno cerebral y un aumento del FSC por vasodilatación, aunque la autorregulación se encuentra preservada siempre que se mantenga una concentración alveolar mínima menor a 1 CAM en el caso del desfluorane y de 1.5 CAM en el caso del sevofluorane. No obstante, esta vasodilatación puede conllevar un "efecto robo" a las regiones irrigadas por vasos EMM, ocasionando isquemia. Sato, et al. ${ }^{5}$ concluyeron que existía una disminución del flujo sanguíneo regional cortical (FSCor) en pacientes sometidos a anestesia inhalatoria en comparación con los sometidos a TIVA durante técnicas de revascularización. Dicha conclusión fue refrendada por Kikuta, et al. ${ }^{6}$, que además demostraron un descenso de la PIC estadísticamente significativo con propofol. Sin embargo, todavía no se han encontrado diferencias significativas en cuanto a la mortalidad, estancia hospitalaria o complicaciones posquirúrgicas entre una técnica u otra. Con respecto a los barbitúricos y su efecto protector cerebral, en nuestro caso, al no realizarse clipaje arterial durante la intervención, no fue necesaria su administración. Lo que sí parece claro es la necesidad de mantener una adecuada analgesia para evitar una respuesta hipertensiva a la intubación y colocación del marco Mayfield, así como la administración de lidocaína en la intubación para disminuir la tos. 
Como hemos mencionado, una vasodilatación cerebral, secundaria a fármacos, a hipotensión o a hipercapnea, provoca un efecto robo que puede comprometer la irrigación de los tejidos afectos por EMM. De igual manera, una vasoconstricción secundaria a hipertensión o hipocapnea puede originar una isquemia cerebral y, además, puede desencadenar una rotura de los vasos colaterales y, en consecuencia, un evento hemorrágico. Por ello conservar la estabilidad hemodinámica se convierte en un objetivo primordial. En nuestro caso consideramos admisible una variación de un $20 \%$ de la PAM con respecto a la basal. Para mantenernos en dicho rango, a parte de conservar una adecuada fluidoterapia, administramos fenilefrina, inicialmente en bolos y posteriormente en perfusión continua, sin incidencias.

Por otro lado, el $\mathrm{CO}_{2}$ arterial supone uno de los estímulos más importantes para los vasos cerebrales. Samagh, et al. ${ }^{7}$ demostraron que tanto los pacientes que habían mantenido $\mathrm{EtCO}_{2}$ intraoperatorio por debajo de $31 \mathrm{mmHg}$ como los que habían superado los $35 \mathrm{mmHg}$ tenían una estancia hospitalaria más prolongada que aquellos en los que el $\mathrm{EtCO}_{2}$ permaneció entre 31 y $35 \mathrm{mmHg}$. En otro estudio, Chiu, et al. ${ }^{8}$ demostraron que los pacientes hiperventilados con $\mathrm{EtCO}_{2}$ $<29 \mathrm{mmHg}$ presentaban un descenso del FSCor. Por lo que el objetivo de la ventilación es la normocapnea.

La hidratación es un punto esencial para mantener la PPC. Se recomienda un cierto grado de hipervolemia con cristaloides balanceados siempre conservando un hematocrito por encima de 35\%, y así evitar el uso de manitol como medida antiedema ${ }^{3}$. En su estudio Sato, et al. ${ }^{9}$ ponen de manifiesto como pacientes que han presentado una diuresis cercana a $2 \mathrm{ml} / \mathrm{kg} / \mathrm{h}$ cuentan con mayor morbilidad perioperatoria que aquellos que la han mantenido por encima de $4 \mathrm{ml} / \mathrm{kg} / \mathrm{h}$. Nuestro paciente no presentaba contraindicaciones cardiológicas que evitaran buscar este cierto grado de hipervolemia, por ello nuestra fluidoterapia fue liberal con diuresis horaria elevada.

\section{Conclusiones}

La EMM conlleva alteraciones fisiopatológicas que pueden comprometer la perfusión cerebral, dado que los vasos afectos han perdido la autorregulación. Por ello es imprescindible evitar cambios hemodinámicos bruscos, haciendo uso de fármacos vasoactivos si es necesario. Con respecto a la técnica anestésica, se requieren más estudios para poder recomendar una sobre otra, aunque la TIVA con propofol ha demostrado mantener un mejor flujo regional cortical en comparación con los agentes halogenados.

\section{Responsabilidades éticas}

Protección de personas y animales. Los autores declaran que para esta investigación no se han realizado experimentos en seres humanos ni en animales.

Confidencialidad de los datos. Los autores declaran que en este artículo no aparecen datos de pacientes.

Derecho a la privacidad y consentimiento informado. Los autores declaran que en este artículo no aparecen datos de pacientes.

\section{Bibliografía}

1. Han JS, Abou-Hamden A, Mandell DM, Poublanc J, Crawley AP, Fisher JA, et al. Impact of Extracranial-Intracranial Bypass on Cerebrovascular Reactivity and Clinical Outcome in Patients With Symptomatic Moyamoya Vasculopathy. Stroke. 2011;42:3047-54.

2. García-Fernández E, Pruneda González L, Margolles-Martins M. Enfermedad de moyamoya. Enfermedades raras en Asturias. Asturias. Dirección General de Salud Pública Consejería de Sanidad. 2013. Disponible en: https://www.asturias.es/portal/site/astursalud/menuitem.com.

3. Parray T, Martin TW, Siddiqui S. Moyamoya Disease: A Review of the Disease and Anesthetic Management. J Neurosurg Anesthesiol. 2011; 23:100-9.

4. Yáñez L, Paredes M, Lapadula M, Martínez P, Durán F. Enfermedad de Moyamoya, a propósito de dos casos. Rev Chil Pediatr. 2008;79(6):629-35.

5. Sato $K$, Shirane $R$, Kato $M$, et al. Effect of inhalation anesthesia on cerebral circulation in moyamoya disease. J Neurosurg Anesth. 1999;11:25-30.

6. Kikuta K, Takagi Y, Nozaki K, et al. Effects of intravenous anesthesia with propofol on regional cortical blood flow and intracranial pressure in surgery for moyamoya disease. Surgical Neurol. 2007:68:421-4.

7. Samagh N, Bhagat H, Grover VK, Sahni N, Agarwal A, Gupta SK. Retrospective analysis of perioperative factors on outcome of patients undergoing surgery for Moyamoya disease. J Neurosc Rural Prac. 2015;6:262-5.

8. Chiu D, Shedden P, Bratina P, Grotta JC. Clinical features of Moyamoya disease in the United States. Stroke. 1998;29:1347-51.

9. Sato K Shirane R, Yoshimoto T. Perioperative factors related to the development of ischemic complications in patients with moyamoya disease. Childs Nerv System. 1997;13:68-72 